\title{
Experimental investigation of an unusual induction effect and its interpretation as a necessary consequence of Weber electrodynamics
}

This paper was downloaded from TechRxiv (https://www.techrxiv.org).

\section{LICENSE}

CC BY 4.0

SUBMISSION DATE / POSTED DATE

30-08-2021 / 07-09-2021

\section{CITATION}

Kühn, Steffen (2021): Experimental investigation of an unusual induction effect and its interpretation as a necessary consequence of Weber electrodynamics. TechRxiv. Preprint.

https://doi.org/10.36227/techrxiv.16535331.v1

$\mathrm{DOI}$

10.36227/techrxiv.16535331.v1 


\title{
Experimental investigation of an unusual induction effect and its interpretation as a necessary consequence of Weber electrodynamics
}

\author{
Steffen Kühn \\ August 30, 2021
}

\begin{abstract}
The magnetic force acts exclusively perpendicular to the direction of motion of a test charge, whereas the electric force does not depend on the velocity of the charge. This article provides experimental evidence that, in addition to these two forces, there is a third electromagnetic force that (i) is proportional to the velocity of the test charge and (ii) acts parallel to the direction of motion rather than perpendicular. This force cannot be explained by the Maxwell equations and the Lorentz force, since it is mathematically incompatible with this framework. However, this force is compatible with Weber electrodynamics and Ampère's original force law, as this older form of electrodynamics not only predicts the existence of such a force but also makes it possible to accurately calculate the strength of this force.
\end{abstract}

\section{Contents}

\section{Introduction}

II Experiment

II-A Concept .............

II-B Implementation ..........

II-C Results . . . . . . . . . . .

\section{Theory}

III-A Current in the transmitter . . . . . . .

III-B Force caused by a current element . . . 5

III-C Induced voltage in the tube ..... 5

III-D Objections ........... 6

IV Summary and conclusion

V Acknowledgements

References

\section{INTRODUCTION}

As generally accepted, the electromagnetic force $\boldsymbol{F}$ onto a test charge $q$ with velocity $\boldsymbol{v}$ is fully given by the two fields $\boldsymbol{E}$ and $\boldsymbol{B}$ and the formula of the Lorentz force

$$
\boldsymbol{F}=q \boldsymbol{E}+q \boldsymbol{v} \times \boldsymbol{B} .
$$

As seen in Equation 1, the formula of the Lorentz force cannot express a force component proportional to $q \boldsymbol{v}$, because (i) $q \boldsymbol{E}$ is independent of $\boldsymbol{v}$ and (ii) the term $\boldsymbol{v} \times \boldsymbol{B}$ is always oriented perpendicular to $\boldsymbol{v}$ because the scalar product $\boldsymbol{v} \cdot(\boldsymbol{v} \times \boldsymbol{B})$ equals zero. Thus, should there be an electromagnetic force that is proportional to the speed of the test charge and acts parallel to the direction of motion, it would be intrinsically incompatible with the Lorentz force (1).
However, the experiment performed in this article provides evidence that such a force exists. Although the existence of this force may seem highly implausible after more than a century of practical experience in electrical engineering, these forces only occur in very special situations. Moreover, there has been little reason to study or search for unexpected effects in century-old theories. Despite the rarity of this force, it is not the first time that such a force has been reported [7]. AndréMarie Ampère himself explicitly investigated this kind of force experimentally and included the results in his force formula [6], [4], [1].

With the present theory of electrodynamics, i.e. Maxwell's equations in combination with the Lorentz force, such a force is incompatible. However, it can be considered in the context of Weber electrodynamics, which is an earlier theory. The term electrodynamics is however a bit misleading for Weber's theory because Weber electrodynamics consists of only a single formula that resembles Coulomb's law. But, in contrast to Coulomb's law, Weber's formula contains not only the distance between the charges as a parameter but also the relative speed and acceleration of the charges.

Because Weber electrodynamics is only a force law without fields, it is not suitable for explaining electromagnetic waves. This is not a principle-related deficiency, but rather a result of the fact that Weber electrodynamics was almost completely forgotten following the success of the Maxwell equations. It could even be argued that it was simply missed to find the corresponding field equations. Apart from this obvious shortcoming, Weber electrodynamics is a remarkably simple and powerful theory that can presumably explain all quasistationary effects [8], [3].

The simplest way to understand Weber electrodynamics from a modern point of view is to assume that the potential energy between two point charges $q_{s}$ and $q_{d}$ at the locations $\boldsymbol{r}_{s}$ and $\boldsymbol{r}_{d}$, respectively, is given by the formula

$$
V=\frac{1}{\gamma(\dot{r})} V_{C}(r),
$$

where

$$
V_{C}(r)=\frac{q_{s} q_{d}}{4 \pi \epsilon_{0} r}
$$

is the classical potential energy of two point charges at rest with respect to each other. In this formula, $r:=\|\boldsymbol{r}\|$ is the distance between the two point charges; that is, the Euclidean norm of the distance vector

$$
\boldsymbol{r}=\boldsymbol{r}_{d}-\boldsymbol{r}_{s}
$$


As per convention, the dot on top of a symbol indicates the derivative with respect to time. Therefore, $\dot{r}=\dot{\boldsymbol{r}} \cdot \boldsymbol{r} / \boldsymbol{r}$ is not the differential velocity $\dot{\boldsymbol{r}}=\dot{\boldsymbol{r}}_{d}-\dot{\boldsymbol{r}}_{s}$ nor its Euclidean norm. $\dot{r}$ is instead the relative speed, i.e., the speed with which the two charges approach or move away from each other on their connecting line.

$\gamma$ is the Lorentz factor known from special relativity. If the relative speed $\dot{r}$ between the two point charges is zero, the Lorentz factor is equal to one, and Equation (2) becomes the usual formula for the potential energy of a resting point charge in the field of another resting point charge (3).

For small relative speeds $\dot{r}$, which can be verified by calculating the Taylor series, the approximation

$$
V \approx\left(1-\frac{\dot{r}^{2}}{2 c^{2}}\right) \frac{q_{s} q_{d}}{4 \pi \epsilon_{0} r}
$$

can be obtained. This formula appears for the first time in 1848 in a publication by Wilhelm Weber [3]. The corresponding force formula for the potential energy described by Equation (5) is

$$
\boldsymbol{F}=\frac{q_{s} q_{d}}{4 \pi \epsilon_{0}} \frac{\boldsymbol{r}}{r^{3}}\left(1-\frac{\dot{r}^{2}}{2 c^{2}}+\frac{r \ddot{r}}{c^{2}}\right) .
$$

This force formula dates back to 1846 [3]. The relationship between the Weber force (6) and potential energy (5) can be verified in a few steps and is given by

$$
-\dot{V}=\boldsymbol{F} \cdot \dot{\boldsymbol{r}}
$$

which is an alternative representation of the law of energy conservation, as the term on the right side represents the time derivative of the kinetic energy.

Equation (6) expresses the force between two electric point charges. However, most macroscopic considerations involve electric currents. An electric current is a multi-particle phenomenon. For example, a current could be a metal wire with positively charged ions at rest and negatively charged electrons moving at a non-zero average velocity.

A unique feature of Weber's force law is that the original form of Ampère's force law can be derived from Weber's law without additional assumptions [2]. This means that the Weber force is a microscopic explanation of the magnetic forces between arbitrarily shaped conductor loops. This interpretation is noteworthy given the simplicity of the potential energy expression (2), it suggests that one could work without a vector potential, magnetic field, or Lorentz force (1).

It should also be noted that the Weber force satisfies the conservation laws for momentum, angular momentum, and energy. The Liénard-Schwarzschild force (equation (8) in reference [3]), which is the counterpart to Weber's force that follows from Maxwell's equations, violates these conservation laws and therefore seems implausible.

The next section of this article describes an experiment in which an unusual aspect of Weber electrodynamics comes into play. The third section provides a detailed theoretical analysis of this unusual effect.

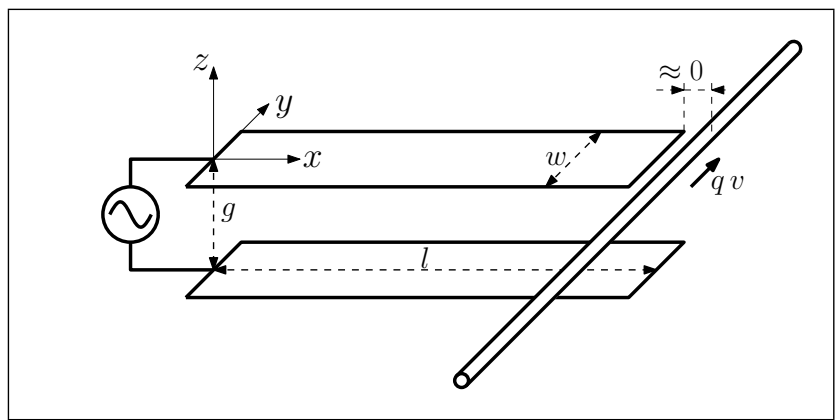

Figure 1. The principle of the experiment is to determine whether, during the charging and discharging of a capacitor (left), a force is generated on fastmoving charge carriers in a tube (right), and whether this force is proportional to the speed of the charge carriers and acts in their direction of motion.

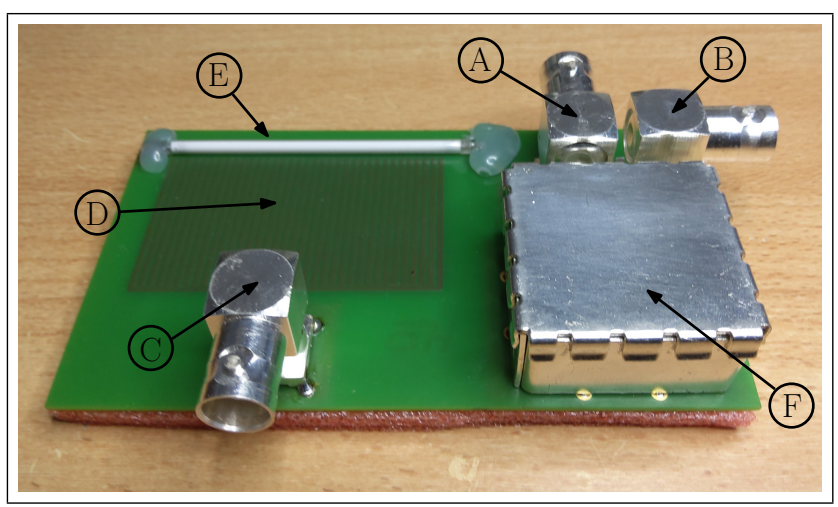

Figure 2. Circuit board: (A) Connection for a high DC voltage to operate the tube; (B) measuring connector; (C) input feed to the transmit antenna; (D) capacitor as the transmit antenna, $(\mathrm{E})$ receiver tube, $(\mathrm{F})$ shielded receiver circuit.

\section{EXPERIMENT}

\section{A. Concept}

The basic concept of this experiment is to determine whether fast-moving charge carriers that are moving sideways past the plate of a capacitor perceive a force in their direction of motion when the plate capacitor is charged and discharged by an alternating current. Figure 1 shows the principle of the experiment.

As the sketch in Figure 1 suggests, the experiment is conducted by placing a long tube with fast-moving electrons near one of the plates of a plate capacitor. The capacitor (left side of Figure 1) acts as an antenna and radiates an electromagnetic wave. The exact shape of the electromagnetic wave is not relevant for this experiment because neither the magnetic nor the electric field are able to generate a force or voltage in the tube, which would be proportional to the speed of the charge carriers in the tube.

\section{B. Implementation}

The experiment was performed using a $6.0 \mathrm{~cm} \times 10.5 \mathrm{~cm}$ double-layer printed circuit board (PCB) that was $1 \mathrm{~mm}$ thick and made of FR4. Figure (2) is a photograph of the exact circuit board used in this experiment. 


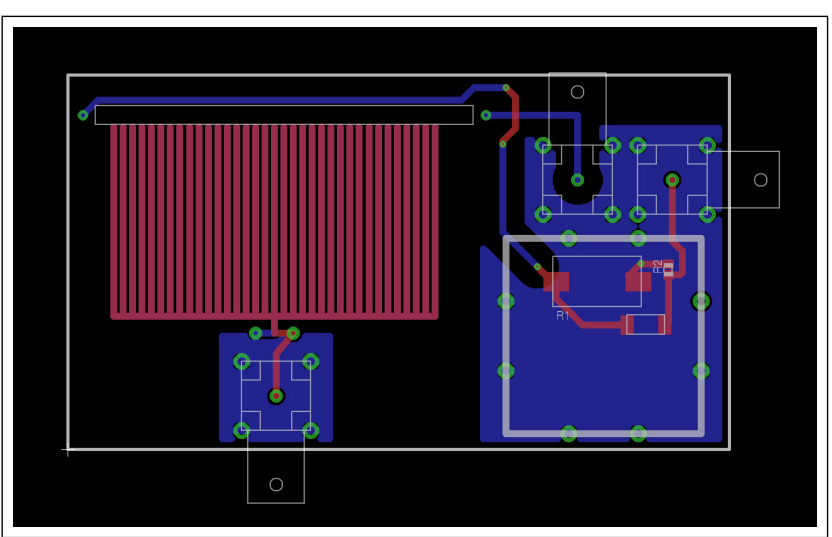

Figure 3. Printed circuit board with receiver and transmit capacitor. The top side is shown in red, and the bottom side is shown in blue

As shown in Figure 2, there are three BNC connectors on the board. The first BNC connector (A) is for connecting a $900 \mathrm{~V}$ DC voltage to operate the tube. Socket (B) is the connection for the oscilloscope, and socket (C) is for connecting a waveform generator to capacitor (D). The capacitor is comb-shaped both on top and bottom of the board and has a capacitance of $37 \mathrm{pF}$. The reasons for choosing this shape of capacitor will be described in the theory section of this article. The metal case $(\mathrm{F})$ in Figure 2 contains a few components that are shielded against electromagnetic interference. The ground of the housing is connected to the ground for sockets (A) and (B), but not with the ground for socket (C).

The receiver (E) is a type BF2661-24B cold-cathode fluorescent lamp (CCFL) from the manufacturer JKL. The tube emits UV radiation at a wavelength of $\lambda=253.7 \mathrm{~nm}$ [9]. A CCFL is a type of tube in which the electrons are only drawn from the cathode by the high intensity of the electric field. Because the tube contains a gas rather than a vacuum, the speed $v$ of the electrons is not proportional to the applied voltage and can therefore only be estimated to be approximately $0.76 \%$ of the speed of light in a vacuum, $c$, using the following equation:

$$
\frac{1}{2} m_{e} v^{2}=h \frac{c}{\lambda}
$$

where $m_{e}$ is the mass of the electron and $h$ is Planck's constant. This experiment used a CCFL tube because the external dimensions imposed tight constraints and a sufficiently thin tube with a Wehnelt cylinder and without gas filling was not available as a component.

Figure 3 shows the layout of the two-layer PCB, with the top layer shown in red and the bottom layer in blue. The corresponding circuit, without the capacitor (D) serving as the transmit antenna, is shown in Figure 4. The circuit consists only of a load resistor R1, which limits the current through the tube to $1.39 \mathrm{~mA}$, and a passive high-pass filter. The high-pass filter consists of a high-voltage capacitor $\mathrm{C} 1$ and a resistor $\mathrm{R} 2$ that decouple the measurement connector (B) from the high voltage and filters out frequencies below $\approx 1 \mathrm{MHz}$.

Components R1, R2, and $\mathrm{C} 1$ are located under a shielded metal housing and above a ground plane on the bottom side of the

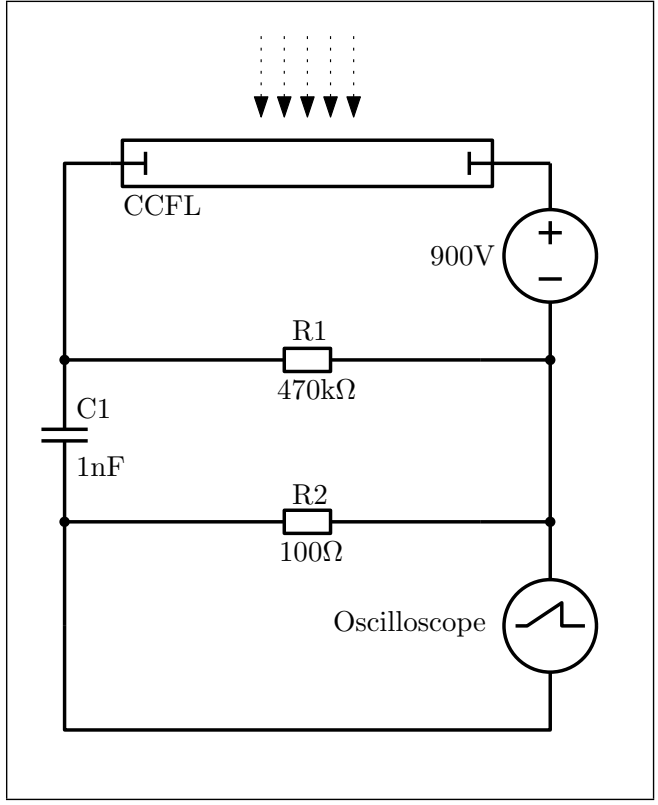

Figure 4. Circuit of the receiver, shown without the transmit capacitor

PCB. The traces outside the housing were designed to be as short as possible. The area of the receiving antenna's conductor loop was minimized; however, some compromises had to be made with this design, as the high voltage imposed minimum distances.

\section{Results}

The first measurement of the experiment was performed without the tube soldered in. This was done to determine how strongly the feed line of the tube would act as an electrical antenna for parasitic longitudinal electric fields. For this purpose, a sinusoidal voltage with an amplitude of $2.5 \mathrm{~V}$ and a frequency of $20-60 \mathrm{MHz}$ was applied to the BNC socket (C). The result is shown in Figure 5 as a black solid line (A). In this experiment, it turned out that it made no difference whether the high voltage was on or off, as the measured curve was almost identical in both cases. The amplitude of the measured signal frequency was also two to three orders of magnitude higher than that of interfering frequencies and was therefore clearly distinguishable.

After the tube was installed, the experiment was repeated twice, once with the high voltage source off and once with it on. The results are shown as curves (B) and (C) in Figure 5, where curve (B) was measured with the high voltage switched off. Even with the tube turned off, the amplitude generally increased by about $1.5 \mathrm{mV}$ compared to curve (A) measured without the tube present. This would be expected, as the gas in the tube can be polarized and therefore reacts to parasitic electric fields in the longitudinal direction of the tube.

The amplitude was further increased when the high voltage was switched on (i.e., when the electrons in the tube were moving with a speed of approximately $0.0076 c$ ). This is remarkable and cannot be explained by the longitudinal electric field component, as the electric force does not depend on 


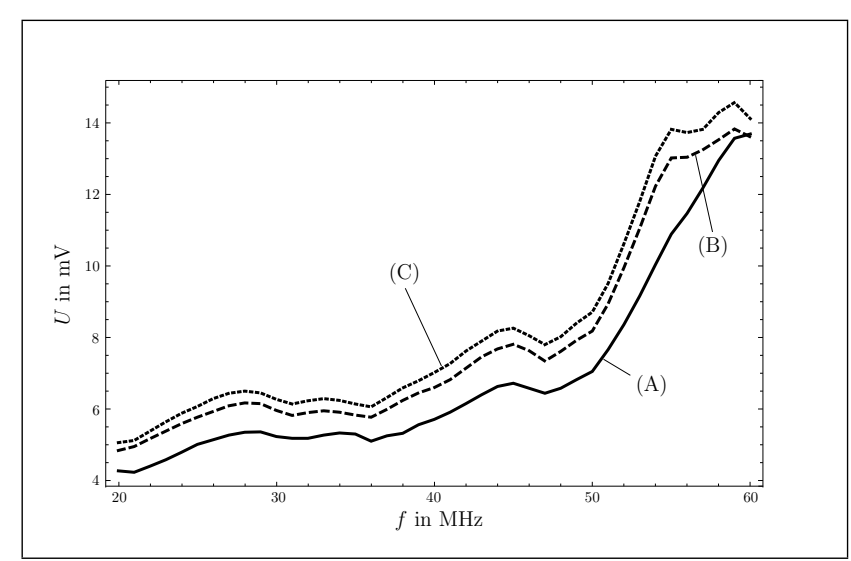

Figure 5. Measured amplitudes as a function of the transmission frequency: (A) without tube; (B) with tube but without high voltage; (C) with tube and high voltage.

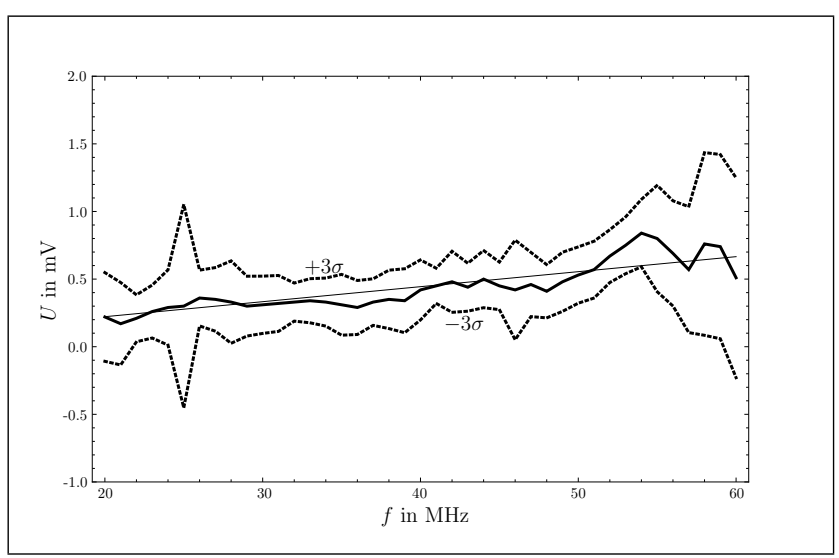

Figure 6. The measured voltage difference between the experiments run with high voltage switched off and on. The dashed curves indicate the $3 \sigma$ confidence interval. The thin line is a linear fit of the measured curve. The slope of the line is $11.1 \mathrm{pV} / \mathrm{Hz}$.

the speed of the charge carriers and a magnetic field cannot accelerate charge carriers in the direction of motion.

If we calculate the difference between the curves (C) and (B), we obtain the curve shown in Figure 6, which shows a linear frequency dependence. This is consistent with Weber electrodynamics, as will become evident in the theory section.

\section{THEORY}

\section{A. Current in the transmitter}

To analyze the experimental results, we first need to find the current $i(x, t)$ in the transmit capacitor (D) in Figure 2. As shown in Figure 1, a single tooth of the comb-shaped capacitor can be schematically interpreted as a biplanar microstrip with a sinusoidal AC voltage $u(t)=u(x=0, t)=U_{0} e^{\mathrm{i} \omega t}$, amplitude $U_{0}$, and angular frequency $\omega$ applied to its input at $x=0$.

To calculate the current, we consider the microstrip to be an unterminated transmission line. The telegrapher's equations can be applied in this context, and it is therefore possible to use Equation (15) from reference [10] to calculate the transfer function. Because the transmission line is unterminated in this example, the termination impedance is $Z_{T} \rightarrow \infty$. Equation (15) from reference [10] therefore simplifies to

$$
H=\frac{\cosh \left((l-x) \sqrt{\frac{Z_{L}^{\prime}}{Z_{Q}^{\prime}}}\right)}{\cosh \left(l \sqrt{\frac{Z_{L}^{\prime}}{Z_{Q}^{\prime}}}\right)} .
$$

In this equation,

$$
Z_{L}^{\prime}=\mathrm{i} \omega L^{\prime}
$$

is the series impedance, with $L^{\prime}$ being the inductance per meter, and

$$
Z_{Q}^{\prime}=\frac{1}{\mathrm{i} \omega C^{\prime}},
$$

where $C^{\prime}$ is the capacitance per meter. The series resistance is neglected as irrelevant.

Equation (16) from reference [10] defines the voltage

$$
u(x, t)=H U_{0} e^{\mathrm{i} \omega t}
$$

along the microstrip, and Equation (20) gives the current

$$
i(x, t)=-\frac{1}{Z_{L}^{\prime}} \frac{\partial H}{\partial x} U_{0} e^{\mathrm{i} \omega t} .
$$

Substituting Equation (9) into these expressions gives

$$
u(x, t)=\frac{\cos \left(\sqrt{L^{\prime} C^{\prime}} \omega(l-x)\right)}{\cos \left(\sqrt{L^{\prime} C^{\prime}} \omega l\right)} U_{0} e^{i \omega t}
$$

and

$$
i(x, t)=\sqrt{\frac{C^{\prime}}{L^{\prime}}} \frac{\sin \left(\sqrt{L^{\prime} C^{\prime}} \omega(l-x)\right)}{\cos \left(\sqrt{L^{\prime} C^{\prime}} \omega l\right)} U_{0} e^{\mathrm{i}\left(\omega t+\frac{\pi}{2}\right)} .
$$

The maximum frequency in the experiment was $60 \mathrm{MHz}$, which corresponds to a wavelength of $5 \mathrm{~m}$. Because this is a long wavelength relative to the length $l=3 \mathrm{~cm}$ of the capacitor, the voltage (14) and current (15) can be approximated using the first order Taylor series with respect to $\omega$. This simplifies Eqs. (14) and (15) to

$$
u(x, t) \approx u(t)=U_{0} e^{\mathrm{i} \omega t}
$$

and

$$
i(x, t) \approx C^{\prime} \omega(l-x) U_{0} e^{\mathrm{i}\left(\omega t+\frac{\pi}{2}\right)} .
$$

As these equations show, the voltage everywhere in the capacitor is equal to the input voltage. However, as expected, the current precedes the voltage by $90^{\circ}$, decreases linearly and disappears completely by the end of the line. The inductance per meter $L^{\prime}$ has no effect. Because the experiment uses a simple plate capacitor, the capacitance per meter $C^{\prime}$ can be defined as

$$
C^{\prime}=\frac{\epsilon_{r} \epsilon_{0} w}{g}
$$

where $\epsilon_{r}$ is the relative permittivity of the medium between the plates. Using the parameters of the capacitor in our experiment $\left(\epsilon_{r}=4, w=1 \mathrm{~mm}\right.$ [width of one of the 35 teeth] and $g=$ $1 \mathrm{~mm}$ ), we obtain $C^{\prime}=35.4 \mathrm{pF} / \mathrm{m}$. 


\section{B. Force caused by a current element}

The current in a metallic wire consists of electrons moving with a drift velocity $\boldsymbol{u}$ while metal ions, which compensate the negative charge of the electrons towards the outside of the wire, are at rest. The force exerted by a short segment of a wire of length $\zeta$ at the origin of the coordinate system onto a test charge $q$ is therefore equal to the sum of the Weber forces of all resting metal ions and all moving electrons in the wire.

We will now calculate this force using the equations derived above. First, the formula of the Weber force (6) must be converted into a more practicable form. Let $\boldsymbol{v}:=\dot{\boldsymbol{r}}$ be the first time derivative of the distance vector (4) and $\ddot{\boldsymbol{r}}:=\boldsymbol{a}=\dot{\boldsymbol{v}}$ be the second derivative. This allows us to set up the equations

$$
\dot{r}=\frac{\mathrm{d}}{\mathrm{d} t} \sqrt{\boldsymbol{r} \cdot \boldsymbol{r}}=\frac{\boldsymbol{r} \cdot \boldsymbol{v}}{r}
$$

and

$$
\ddot{r}=\frac{\mathrm{d}}{\mathrm{d} t} \dot{r}=\frac{v^{2}}{r}+\frac{\boldsymbol{r} \cdot \boldsymbol{a}}{r}-\frac{(\boldsymbol{r} \cdot \boldsymbol{v})^{2}}{r^{3}} .
$$

Substituting these two equations into the Weber force equation (6) for $\boldsymbol{a} \approx \mathbf{0}$, we obtain the following force formula in vector notation:

$$
\boldsymbol{F}\left(q_{s}, q_{d}, \boldsymbol{r}, \boldsymbol{v}\right)=\left(1+\frac{v^{2}}{c^{2}}-\frac{3}{2}\left(\frac{\boldsymbol{r}}{r} \frac{\boldsymbol{v}}{c}\right)^{2}\right) \frac{q_{s} q_{d}}{4 \pi \epsilon_{0}} \frac{\boldsymbol{r}}{r^{3}} .
$$

Assume that there are $n$ electrons moving in the piece of wire. The total force $\boldsymbol{F}_{T}$ of the wire segment onto a test charge $q$ moving with a velocity $\boldsymbol{v}$ at location $\boldsymbol{r}$ is therefore equal to

$$
\begin{aligned}
\boldsymbol{F}_{T} & =\boldsymbol{F}(-n e, q, \boldsymbol{r}, \boldsymbol{v}-\boldsymbol{u})+\boldsymbol{F}(n e, q, \boldsymbol{r}, \boldsymbol{v}) \\
& =\frac{e n q}{8 c^{2} \epsilon_{0} \pi} \frac{\boldsymbol{r}}{r^{3}}\left(3 \frac{(\boldsymbol{u} \boldsymbol{r})^{2}}{r^{2}}-2 u^{2}+4 \boldsymbol{u} \boldsymbol{v}-6 \frac{(\boldsymbol{u r})(\boldsymbol{v} \boldsymbol{r})}{r^{2}}\right) .
\end{aligned}
$$

Because the drift velocities $\boldsymbol{u}$ in metallic conductors are very small, all terms of order $O\left(u^{2}\right)$ can be neglected and, based on the relation $\mu_{0}=1 /\left(c^{2} \epsilon_{0}\right)$ and $\boldsymbol{i}:=-n e \boldsymbol{u} / \zeta$, we can obtain the approximation

$$
\boldsymbol{F}_{T}(\boldsymbol{r}, \boldsymbol{i}) \approx \frac{q \mu_{0} \zeta}{4 \pi} \frac{\boldsymbol{r}}{r^{3}}\left(3 \frac{(\boldsymbol{i r})(\boldsymbol{v r})}{r^{2}}-2 \boldsymbol{i v}\right) .
$$

This approximation corresponds to Ampère's original force law from 1822 [4], but not to the Biot-Savart law in combination with the Lorentz force [7].

\section{Induced voltage in the tube}

The formula (23) derived above can now be used to calculate the force that the total current (17) in the two strip lines of Figure 1 produces on the test charge $q$ in the tube next to the capacitor.

For simplicity, we assume that the strip lines are sufficiently thin that the current can be considered to be a line current. In this case, the force $\boldsymbol{F}_{u}$ of the upper microstrip on a charge $q$ at location $\boldsymbol{r}$ is

$$
\boldsymbol{F}_{u}=\frac{1}{\zeta} \int_{0}^{l} \boldsymbol{F}_{T}\left(\boldsymbol{r}-x \boldsymbol{e}_{x}, i(x) \boldsymbol{e}_{x}\right) \mathrm{d} x .
$$

Because the test charges are only located inside the tube, $\boldsymbol{r}=$ $l \boldsymbol{e}_{x}+y \boldsymbol{e}_{y}$, with $y$ being the only variable parameter. The force $\boldsymbol{F}_{l}$ of the lower microstrip can be calculated analogously to the force of the upper microstrip, giving

$$
\boldsymbol{F}_{l}=\frac{1}{\zeta} \int_{0}^{l} \boldsymbol{F}_{T}\left(\boldsymbol{r}-\left(x \boldsymbol{e}_{x}-g \boldsymbol{e}_{z}\right),-i(x) \boldsymbol{e}_{x}\right) \mathrm{d} x .
$$

It should be noted that the lower microstrip is not only shifted downward by $g$, but that the current also flows in the opposite direction.

The total force $\boldsymbol{F}=\boldsymbol{F}_{u}+\boldsymbol{F}_{l}$ onto the test charge is the sum of the forces of the upper and lower strip lines. Inserting the current (17) and solving the resulting integrals gives the $y$ component of the total force:

$$
\boldsymbol{F} \boldsymbol{e}_{y}=\frac{1}{4 \pi} C^{\prime} l^{3} \mu_{0} q U_{0} v \omega \alpha(y) e^{\mathrm{i}\left(\omega t+\frac{\pi}{2}\right)} .
$$

The auxiliary function $\alpha(y)$ was introduced for readability and is defined as

$$
\alpha(y):=\frac{1}{\left(l^{2}+y^{2}\right)^{3 / 2}}-\frac{y^{2}}{\left(g^{2}+y^{2}\right)\left(g^{2}+l^{2}+y^{2}\right)^{3 / 2}} .
$$

If a charge $q$ is guided along the tube with speed $v$, work is performed on this charge. Electric voltage $U$ is defined as work per charge and can therefore be calculated by solving the integral

$$
U=\frac{1}{q} \int_{-\infty}^{+\infty} \boldsymbol{F} \boldsymbol{e}_{y} \mathrm{~d} y .
$$

The choice of the integration limits can be justified by the fact that work is performed only in the vicinity of the microstrip, and integration at greater distances makes essentially no contribution.

Substituting Equation (26) follows

$$
U=\frac{1}{4 \pi} C^{\prime} l^{3} \mu_{0} U_{0} v \omega e^{\mathrm{i}\left(\omega t+\frac{\pi}{2}\right)} \int_{-\infty}^{+\infty} \alpha(y) \mathrm{d} y .
$$

The calculation of the resulting integral is straightforward and gives

$$
\int_{-\infty}^{+\infty} \alpha(y) \mathrm{d} y=\frac{2 g}{l^{3}} \arccos \left(\frac{g}{\sqrt{g^{2}+l^{2}}}\right) \approx \frac{g \pi}{l^{3}},
$$

with the approximation being valid when $g$ is significantly smaller than $l$, as was the case in our experiment.

If we now substitute this into Equation (29) and compute the absolute value, we obtain the amplitude of the induced voltage:

$$
\hat{U}=\frac{1}{4} C^{\prime} g \mu_{0} U_{0} v \omega .
$$

This equation suggests that, if Ampère's force law is valid in its original form, there must also be a small AC voltage of amplitude $\hat{U}$ that is induced by the capacitor and depends linearly on both the speed $v$ of the electrons and the angular frequency $\omega=2 \pi f$ of the transmitter. This AC voltage 
operates in addition to the high DC voltage that accelerates the electrons in the tube. However, if the Lorentz force (1) is valid, this $\mathrm{AC}$ voltage must not exist.

In total, the capacitor had 35 teeth. Taking this into account and substituting the other experimental parameters

- $C^{\prime}=35.4 \mathrm{pF} / \mathrm{m}$

- $g=1 \mathrm{~mm}$

- $U_{0}=2.5 \mathrm{~V}$

- $v \approx 0.0076 c \approx 2280000 \mathrm{~m} / \mathrm{s}$

into Equation (31), we find that the tube acts like an additional voltage source with an amplitude of approximately $13.9 \mathrm{pV} / \mathrm{Hz}$. This corresponds to a voltage of $0.28 \mathrm{mV}$ at a frequency of $20 \mathrm{MHz}$ and $0.83 \mathrm{mV}$ at $60 \mathrm{MHz}$.

Given the uncertainties and resulting approximations for the properties of the CCFL tube used in this experiment, these calculated voltages agree surprisingly well with the measured results from the experiment. The function estimated from the measured data was $\hat{U}(f) \approx 11.1 \mathrm{pV} / \mathrm{Hz} \cdot f$, which is close to the theoretically estimated value and represents a relative error of only 0.2. This error is even further reduced if the length of the tube and the lateral displacements of the microstrips relative to the tube are also considered.

\section{Objections}

This section evaluates whether the measured voltage can be explained without Weber electrodynamics.

A potential objection to the results of this experiment is that the electric or magnetic field would force the charge carriers in the tube to follow a slightly curved path. This would increase the distance that the charge carriers travel in the tube and, thus, the resistance. However, this objection can be ruled out as an explanation because path lengthening occurs both when charging and discharging the transmit capacitor. Consequently, this effect would create a signal with a frequency twice as high.

Another valid objection is that the gas is partially ionized when current is flowing in the tube but is not ionized when the current is turned off. The permittivity of the medium in the tube would be expected to be different in both cases, which would affect the sensitivity of the antenna to parasitic electric fields longitudinal to the tube. However, a good conducting medium (e.g., plasma) usually has a lower permittivity than a gas. For this reason, antenna sensitivity would be expected to be lower when current flows, but in this experiment, the measured voltages are increased.

\section{Summary AND CONClusion}

There are two key conclusions from this article.

The first conclusion, which is independent of the experiment described here, is that the original form of Ampère's force law is not fully compatible with the Biot-Savart law and modern electrodynamics because modern electrodynamics, for structural reasons, cannot describe a force component that is both proportional to the speed of the test charge and parallel to the direction of motion [7]. However, it has been mathematically shown that Ampère's original force law does contain such force components. This means that any claim that the Biot-Savart law would be equivalent with the Lorentz force must be clearly rejected for purely formal reasons.

The second conclusion from this article derives from the measured results of the experiment described here, because the results agree remarkably well with the predictions of Weber electrodynamics and Ampère's original force law. However, alternative explanations for these results cannot be completely ruled out. To validate the conclusions presented here, this experiment should be repeated with a type of tube that allows the speed of the electrons to be adjusted. Another option would be to use a superconductor instead of a tube, as the speed of the charge carriers can be varied in superconductors as well.

Because the Maxwell equations currently represent the foundation of modern physics, it would be enormously important to test in future experiments if Weber electrodynamics is superior in the near field - and there was recently another indication that this is the case [5]. It would then be a point of intensive research to determine the extent to which statements that are directly or indirectly derived from the Maxwell equations remain valid. It would also be important to find new field equations that are valid for both the near and far fields. For these reasons, it is important to further investigate this subject experimentally.

\section{Acknowledgements}

The author expresses his sincere thanks to Najib Aouni, who brought to his attention that the induction effect studied in this paper could exist.

\section{REFERENCES}

[1] J. P. M. C. Chaib a and F. M. S. Lima. Resuming ampère's experimental investigation of the validity of newton's third law in electrodynamics. Annales de la Fondation Louis de Broglie, 45(1), 2020.

[2] A. Assis. Deriving Ampere's law from Weber's law. Hadronic Journal, 13:441-451, 1990 .

[3] A. Assis and H. T. Silva. Comparison between Weber's electrodynamics and classical electrodynamics. Pramana, 55:393-404, 2000.

[4] A. K. T. Assis and J. P. M. C. Chaib. Ampère's electrodynamics: Analysis of the meaning and evolution of Ampère's force between current elements, together with a complete translation of his masterpiece: Theory of electrodynamic phenomena, uniquely deduced from experience. C. Roy Keys Inc., 2015.

[5] C. Baumgärtel, Ray T. Smith, and S. Maher. Accurately predicting electron beam deflections in fringing fields of a solenoid. Scientific Reports, 2020.

[6] Peter Graneau. Electromagnetic jet-propulsion in the direction of current flow. Nature, 295(5847):311-312, 1982.

[7] Peter Graneau. Ampere and Lorentz forces. Physics Letters, 107 A(5), 1985.

[8] Hermann Härtel. Electromagnetic induction from a new perspective. European J of Physics Education, 9(2), 2018.

[9] John H. Kahl. Cold cathode fluorescent lamps (CCFL's) - A history and overview. Technical report, JKL Components Corporation, 1997. 
[10] Steffen Kühn. General analytic solution of the telegrapher's equations and the resulting consequences for electrically short transmission lines. Journal of Electromagnetic Analysis and Applications, 12:71-87, 01 2020. 